\title{
Web report burnsurgery.org: a starting point for intensivists
} Neill Adhikari ${ }^{1}$ and Joel S Fish ${ }^{2}$

\author{
${ }^{1}$ Clinical Associate, Department of Medicine and Department of Critical Care Medicine, Sunnybrook and Women's College Health Sciences Centre, \\ Toronto, Ontario, Canada \\ ${ }^{2}$ Medical Director, Ross Tilley Burn Centre, Department of Surgery, Sunnybrook and Women's College Health Sciences Centre, Toronto, Ontario, \\ Canada
}

Correspondence: Neill Adhikari, neill.adhikari@sw.ca

Reported: 19 November 2003

Critical Care 2004, 8:143-144 (DOI 10.1186/cc2414)

Published online: 24 December 2003

This article is online at http://ccforum.com/content/8/2/143

(c) 2004 BioMed Central Ltd (Print ISSN 1364-8535; Online ISSN 1466-609X)

Website burnsurgery.org
URL http://www.burnsurgery.org
Cost Individual subscription: free

Keywords burns, critical care

Intensivists may provide initial management to patients with major burns, despite lacking extensive knowledge and experience. Burnsurgery.org is a free website "designed to provide comprehensive, up-to-date educational tools for burn care professionals throughout the world". The site is operated by a nonprofit educational group, with editors from Brigham and Women's Hospital in Boston, MA, USA. Potential conflicts of interest are not disclosed, although the site is linked to some commercial sites promoting anabolic and wound care products. Like many health information websites, this site does not report certification by any quality rating organization or adherence to any of the available quality codes of conduct [1].

The site is divided into educational modules, beginning with burn prevention. The most useful sections discuss initial management priorities, including airway assessment, fluid resuscitation, and burn wound size and depth assessment. Overall, the information focuses on resuscitation issues relevant to intensivists and is consistent with current practice. The sample admission order set, which is presented with the rationale for specific orders, is a useful reminder of important initial therapies. Two significant content errors are the failure to caution against cutting the endotracheal tube prior to intubation (in order to prevent disappearance of the tube into the mouth when facial edema becomes massive) and the recommendation to administer intravenous fluids via peripheral veins (which are often impossible to access in a major burn injury and are prone to dislodgement during patient transfer). This section also includes special issues in initial wound management, chemical burns, electrical burns, comanagement of burns and major trauma, and indications for transfer to a burn facility.
The site has some limitations. The authors should acknowledge the limited contribution of methodologically robust clinical evidence to most treatment recommendations and the extensive interinstitutional variation in some aspects of care (e.g. wound management). Most of the resuscitation information is not referenced, except for that in the section on burn orders. The discussion of pulmonary issues, such as acute respiratory distress syndrome, is limited and does not incorporate current evidence from the critical care literature. Multiple errors of grammar and spelling distract from the information presented. Although the site appears to be recent, with content posted between 2000 and 2002, the authors do not state the frequency of updating. Finally, it would be very useful to review management recommendations in the context of 'best practices', such as the American Burn Association's practice guidelines for burn care.

The website is reasonably functional. Navigation within the site starts from a detailed table of contents on the home page. Similar pages are unfortunately not linked (e.g. initial management to orders), thus requiring a return to the home page to transfer between different sections. This process often entails using the browser's back button because some pages are not linked to the home page. There are no irritating pop-up advertisements or requests for personal information. Most links to other sites are functional.

Overall, this site provides an excellent starting point for intensivists involved in the resuscitation and stabilization of burn patients, especially in situations where immediate consultation and transfer to a major burn center is not possible. 


\section{Best feature}

Excellent coverage of resuscitation topics.

\section{Worst feature}

No validation of the quality of the information provided, either by adherence to a code of conduct or by third-party certification.

\section{Wish list}

A discussion of recommendations in the context of clinical evidence and American Burn Association practice guidelines, and improved site navigation.

\section{Other links}

Sage diagram - http://www.sagediagram.com/

An online burn size and fluid requirements calculator. Users can download the program to handheld computers running the Palm operating system. This website is free to use.

Practice guidelines -

http://www.ameriburn.org/pub/PracticeGuidelines.htm

Information on obtaining practice guidelines for burn care, developed by the American Burn Association in 2001.

Unfortunately, these are not available online. Nonmembers of the American Burn Association can order a copy for US\$60.

Advanced Burn Life Support Course http://www.ameriburn.org/ABLS/ABLS.htm

Description and schedule of the Advanced Burn Life Support Course. The registration fee for the courses ranges from US\$150 to US\$325.

\section{Competing interests}

None declared.

\section{Reference}

1. Wilson P, Risk A: How to find the good and avoid the bad or ugly: a short guide to tools for rating quality of health information on the internet. Commentary: on the way to quality. $\mathrm{Br}$ Med J 2002, 324:598-602. 